\title{
BMJ Open Is progression-free survival associated with a better health-related quality of life in patients with lung cancer? Evidence from two randomised trials with afatinib
}

\author{
Ingolf Griebsch, ${ }^{1}$ Michael Palmer, ${ }^{2}$ Peter M Fayers, ${ }^{3,4}$ Stuart Ellis ${ }^{5}$
}

To cite: Griebsch I,

Palmer M, Fayers PM, et al. Is progression-free survival associated with a better health-related quality of life in patients with lung cancer? Evidence from two randomised trials with afatinib. BMJ Open 2014;4: e005762. doi:10.1136/ bmjopen-2014-005762

- Prepublication history and additional material is available. To view please visit the journal (http://dx.doi.org/ 10.1136/bmjopen-2014005762)

Received 22 May 2014 Revised 28 July 2014 Accepted 11 August 2014

\section{CrossMark}

${ }^{1}$ Boehringer Ingelheim $\mathrm{GmbH}$, Ingelheim, Germany

${ }^{2}$ Keele University, Keele, UK ${ }^{3}$ Institute of Applied Health Sciences, University of Aberdeen, Scotland, UK ${ }^{4}$ Department of Cancer Research and Molecular Medicine, Norwegian University of Science and Technology (NTNU), Trondheim, Norway ${ }^{5}$ Independent Statistical Consultant, Cheshire, UK

Correspondence to Dr Ingolf Griebsch; ingolf.griebsch@boehringeringelheim.com

\section{ABSTRACT}

Objective: Progression-free survival (PFS) is frequently used as an efficacy end point in oncology clinical trials. However, there is limited evidence to support a positive association between improvement in PFS and improvement in health-related quality of life (HRQoL). The association between PFS and HRQoL was evaluated in two randomised trials.

Materials and methods: Data from two randomised controlled trials in patients with non-small cell lung cancer (NSCLC; LUX-Lung 1 and LUX-Lung 3) were used to investigate $\mathrm{HRQ}$ oL in patients to determine whether tumour progression is accompanied by worsening HRQoL. HRQoL was assessed using the cancer-specific European Organization for Research and Treatment of Cancer (EORTC) core questionnaire QLQ-C30, the EuroQol EQ-5D overall utility and EuroQol EQ visual analogue scale. In both studies, progression was evaluated by independent review using RECIST criteria (primary end point) and also by investigator assessment. The relationship between tumour progression and $\mathrm{HRQ}$ LL was evaluated using analysis of covariance and a longitudinal model.

Results: Compliance with HRQoL questionnaire completion was high. In both studies, patients with progression consistently experienced numerically poorer HRQoL at the time of progression than patients without progression. Differences in mean scores were statistically significant $(p<0.05)$ between patients with and without progression at week 4 in all analyses in LUX-Lung 1 and at multiple time points in LUX-Lung 3. Results from the longitudinal analysis showed that progression (by independent review and investigator assessment) appears to have consistent negative impact on all three $\mathrm{HRQOL}$ measures (all $\mathrm{p}<0.0001$ ). Conclusions: Tumour progression in patients with NSCLC was associated with statistically significant worsening in HRQoL. These findings confirm the value of PFS as a patient-relevant end point.

\section{INTRODUCTION}

Use of progression-free survival (PFS) as a primary end point in oncology has increased

\section{Strengths and limitations of this study}

- A strength of our analyses is that they address methodological limitations of previous studies investigating this association and use data from two different non-small cell lung cancer studies. In addition, HRQoL was measured using validated assessment tools

- Limitations relate to the handling of missing data, inherent in this type of analysis

- Limited data on the health states of patients with progression was available; this is an additional limitation.

recently, as has its use as a secondary end point. ${ }^{1}$ Using PFS, as opposed to overall survival $(\mathrm{OS})$, has several advantages for clinical trial conduct; trials that use PFS as a primary end point can be conducted more quickly and with fewer patients than trials using OS. ${ }^{1}$ This also benefits patients as it allows earlier access to new treatments as trial results are available sooner when PFS is used as an end point. PFS also directly measures the effect of the investigational treatment and, unlike $\mathrm{OS}$, is insensitive to bias from subsequent treatment(s) (ie, treatment received after disease progression has been determined). ${ }^{2}$ This issue of bias in interpretation of OS data is also compounded by the fact that use of subsequent therapies generally differs between treatment arms. ${ }^{2}$

Despite the advantages of PFS, there are several limitations to consider. There are no standard regulatory criteria for defining progression in clinical trials ${ }^{2}$ and progression can be difficult to assess and subject to measurement error and bias, especially if assessors are not blinded to treatment. ${ }^{2}$ PFS is also influenced by frequency of assessment, unlike OS. ${ }^{3}$ Even though an improvement in 
PFS is considered an indication of disease control and stabilisation, ${ }^{4}$ there is still debate as to whether an improvement in PFS is beneficial for patients. ${ }^{5}$ As such, it is important that PFS benefits seen in clinical trials are accompanied by better symptom control, fewer treatment-related adverse events and better health-related quality of life (HRQoL). ${ }^{14}$

While randomised controlled trials (RCTs) frequently assess HRQoL as well as PFS, the design of such trials only allows indirect inferences regarding a relationship between PFS and HRQoL in situations wherein both are influenced by treatment. For this reason, some health technology assessment agencies ${ }^{6}$ do not consider PFS a patient-relevant outcome measurement and usually discard the information on this end point in their evaluations, particularly in indications and for investigational compounds where PFS may not be a well-established surrogate for OS.

Thus, there is a need to establish the relationship between changes in PFS and HRQoL. While many clinical trials include HRQoL assessments as trial outcomes, a major limitation in evaluating this relationship is that HRQoL assessments are often only administered until disease progression based on imaging results (which may precede symptomatic progression) to avoid the confounding effects of subsequent therapies ${ }^{14}$ and to ease administrative burden. A direct comparison of HRQoL in patients who are considered progression-free with those patients who experience tumour growth is often limited.

Several investigators have assessed the relationship between HRQoL and tumour response in patients with breast, colorectal and renal cell cancer, ${ }^{7-10}$ and suggest that patients who remain on treatment and who experience delayed progression have a stable HRQoL or experience a less rapid decline in HRQoL than patients whose tumours are progressing. To the best of our knowledge, no data have been reported in non-small cell lung cancer (NSCLC).

Two RCTs investigated the role of afatinib, an irreversible ErbB Family Blocker, in NSCLC and included assessment of patient-reported symptoms and HRQoL in addition to tumour progression: LUX-Lung 1 (NCT00656136) ${ }^{11} 12$ and LUX-Lung 3 (NCT00949650). ${ }^{13}{ }^{14}$ The analyses reported here use data collected in these trials to investigate HRQoL in patients before and after progression, and to explore the relationship between tumour progression and HRQoL. Two different statistical analysis methods were used in order to assess the strength of the findings.

\section{PATIENTS AND METHODS \\ Study design}

This analysis used data from two RCTs. ${ }^{12}{ }^{14}$ Key details of the methodology and findings of these trials are summarised in table 1.

\section{Health-related quality of life assessment}

HRQoL was assessed using the self-administered cancerspecific European Organization for Research and Treatment of Cancer (EORTC) multidimensional core questionnaire QLQ-C30. ${ }^{15}$ QLQ-C30 comprises of 30 questions of multi-item and single-item measures. Individual items are scored on a four-point scale, while Global health status (question 29) and quality of life (QoL, question 30) are scored on a seven-point scale.

\begin{tabular}{|c|c|c|}
\hline & LUX-Lung 1 ( $\mathrm{N}=585)$ & LUX-Lung $3(\mathrm{~N}=345)$ \\
\hline Inclusion criteria & $\begin{array}{l}\text { Stage IIIB (with pleural effusion) or stage IV } \\
\text { adenocarcinoma according to the TNM } \\
\text { classification system by the UICC } 6 \text { th edition; } \\
\text { ECOG PS 0-2; patients were not screened for } \\
\text { EGFR mutation status }\end{array}$ & $\begin{array}{l}\text { Patients with stage IIIB/IV lung adenocarcinoma } \\
\text { (AJCC Cancer Staging Manual 6th edition); } \\
\text { EGFR mutation positive disease (Therascreen } \\
\text { EGFR 29; Qiagen, Manchester, UK); ECOG PS } \\
0 \text {-1 }\end{array}$ \\
\hline Previous treatment & $\begin{array}{l}\text { One or two lines of cytotoxic chemotherapy (one } \\
\text { of which was platinum-based) and progressive } \\
\text { disease following at least } 12 \text { weeks of treatment } \\
\text { with erlotinib or gefitinib }\end{array}$ & Treatment-naïve for advanced NSCLC \\
\hline Study treatments & $\begin{array}{l}2: 1 \text { randomisation to } 50 \mathrm{mg} \text { afatinib per day plus } \\
\text { best supportive care }(n=390) \text { or placebo plus } \\
\text { best supportive care }(n=195)\end{array}$ & $\begin{array}{l}2: 1 \text { randomisation to } 40 \mathrm{mg} \text { afatinib per day } \\
(\mathrm{n}=230) \text { or up to six cycles of cisplatin/ } \\
\text { pemetrexed chemotherapy at standard dose } \\
(n=115)\end{array}$ \\
\hline Primary endpoint & OS (from date of randomisation to death) & $\begin{array}{l}\text { PFS (as determined by independent blinded } \\
\text { review) }\end{array}$ \\
\hline Secondary endpoints & Tumour response, PFS, HRQoL, adverse events & Tumour response, OS, HRQoL, adverse events \\
\hline $\begin{array}{l}\text { PFS (afatinib vs } \\
\text { control) by } \\
\text { independent review }\end{array}$ & $\begin{array}{l}3.3 \text { vs } 1.1 \text { months, } \mathrm{HR}=0.38,95 \% \mathrm{Cl} 0.31 \text { to } \\
0.48 ; \mathrm{p}<0.0001\end{array}$ & $\begin{array}{l}11.1 \text { vs } 6.9 \text { months, } \mathrm{HR}=0.58 ; 95 \% \mathrm{Cl} 0.43 \text { to } \\
0.78 ; p=0.001\end{array}$ \\
\hline
\end{tabular}


For the purpose of this analysis, the QLQ-C30 Global health status/QoL (composite of QLQ-C30 questions 29 and 30) score was used to evaluate patients' overall selfreported HRQoL. The EuroQol disease-generic questionnaire, comprising the EQ-5D overall utility and EQ-visual analogue scale (VAS), ${ }^{16}$ was used to assess health status. The EQ-5D measures five dimensions of health (mobility, self-care, usual activities, pain/discomfort and anxiety/depression). Utility scores range from 0 to 1 and were calculated from the five EQ-5D item scores using the UK valuation algorithm. ${ }^{17}$ The EQ VAS records the patient's self-rated health status on a vertical graduated (0-100) VAS.

In LUX-Lung 1, HRQoL questionnaires were scheduled at randomisation, two weekly during the first 2 months of treatment and then every 4 weeks. In LUX-Lung 3, HRQoL was assessed at randomisation and every 21 days. For chemotherapy patients, this was on day 1 of each cycle and was delayed if the chemotherapy was delayed. In both studies, HRQoL was also assessed at the end of treatment following treatment discontinuation due to progression or other reasons and during at least one follow-up visit.

The protocol for both studies required that patients completed HRQoL questionnaires in the clinic using an electronic portable data capture tool before they were provided with any test results by their treating physicians or any other health professional.

\section{Assessment of tumour progression}

Tumour assessments were performed by CT or MRI. In LUX-Lung 1, tumour assessments were undertaken at baseline and every 4 weeks until week 12, and then every 8 weeks until disease progression by independent review. In LUX-Lung 3, tumour assessments were undertaken at baseline and every 6 weeks for the first 48 weeks and then every 12 weeks thereafter until disease progression by independent review or start of new anticancer therapy. RECIST criteria were used for independent review, which was conducted by a central imaging group that included radiologist and oncologist reviewers blinded to treatment assignments; investigators also assessed tumour progression based on radiological and clinical assessment in both studies. In LUX-Lung 3, the primary end point was based on independent review. Independent review is regarded as the most conservative approach and is recommended in RECIST guidelines. ${ }^{18} 19$

\section{Statistical analyses}

The statistical methods used in this analysis were prespecified. For the main analyses, randomised treatment groups were combined in order to increase the numbers of patients available. Additional subgroup analyses were conducted to assess consistency of results between groups. To be included in analysis, patients had to have completed a baseline assessment and at least one measurement at the time of tumour progression or follow-up assessment. A HRQoL assessment was considered valid for inclusion in the statistical analysis if it occurred within \pm 7 days of the date of tumour assessment. In the event there was more than one HRQoL assessment, the one nearest the actual tumour assessment date was used. For patients who progressed, only the first HRQoL assessment was used; after that they were censored for future time points. In the longitudinal analysis, all HRQoL assessments at, or after progression, were used but were censored at the start of any new anticancer therapy. Assessments that were carried out after the start of other subsequent cancer treatment following progression were excluded. All analyses were conducted using data from independent review and investigator assessment of tumour progression. Statistical programming was carried out using S-Plus.

\section{Analysis of covariance}

The hypothesis for this analysis was that patients with and without progression at any time would have different average levels of QoL. At each assessment time (weeks 4, 8 and 12 in LUX-Lung 1 and weeks $6,12,18,24,30$ and 36 in LUX-Lung 3), patients who were classified as progressors (having been 'not progressed' at the previous assessment) were compared with patients who were assessed as 'non-progressors' (between-patient analysis). These time points were chosen for analysis based on the availability of sufficient data. Patients who had died since the previous assessment were excluded from the analyses. At each assessment time and for each HRQoL measure (EORTC Global Health/QoL, EQ-5D UK Utility and EQ VAS) change from baseline in HRQoL score between progressors and non-progressors was compared using an analysis of covariance (ANCOVA) model that included covariates for baseline HRQoL score, progression, Eastern Cooperative Oncology Group Performance Status (ECOG PS; 0 vs $\geq 1$ ), gender and randomised treatment. Adjusted mean changes from baseline in HRQoL measures over time for progressors and non-progressors are presented graphically.

Consistency in the effects of progression was examined by expanding the model to include interaction terms between progression/non-progression and baseline HRQoL, ECOG PS, gender and randomised treatment.

\section{Longitudinal analysis}

The effects of progression on HRQoL over time were investigated using a longitudinal mixed-effects growth curve model, ${ }^{20} 21$ which allows for within-patient assessment of change in HRQoL at or after progression. The model allowed the slope of the growth curve to change at predetermined times since randomisation (weeks 2, 4, 8 and 12 for LUX-Lung 1 and weeks 3, 6, 12, 18 for LUX-Lung 3, based on availability of sufficient data). A cut-off was applied to HRQoL data in each study such that assessments were excluded when fewer than approximately $20-30 \%$ patients remained. Each model included the two random effects of intercept and slope (the week variable). The model included terms for week, covariates 
A Independent review

Week 0

Week 8

Week 12

B

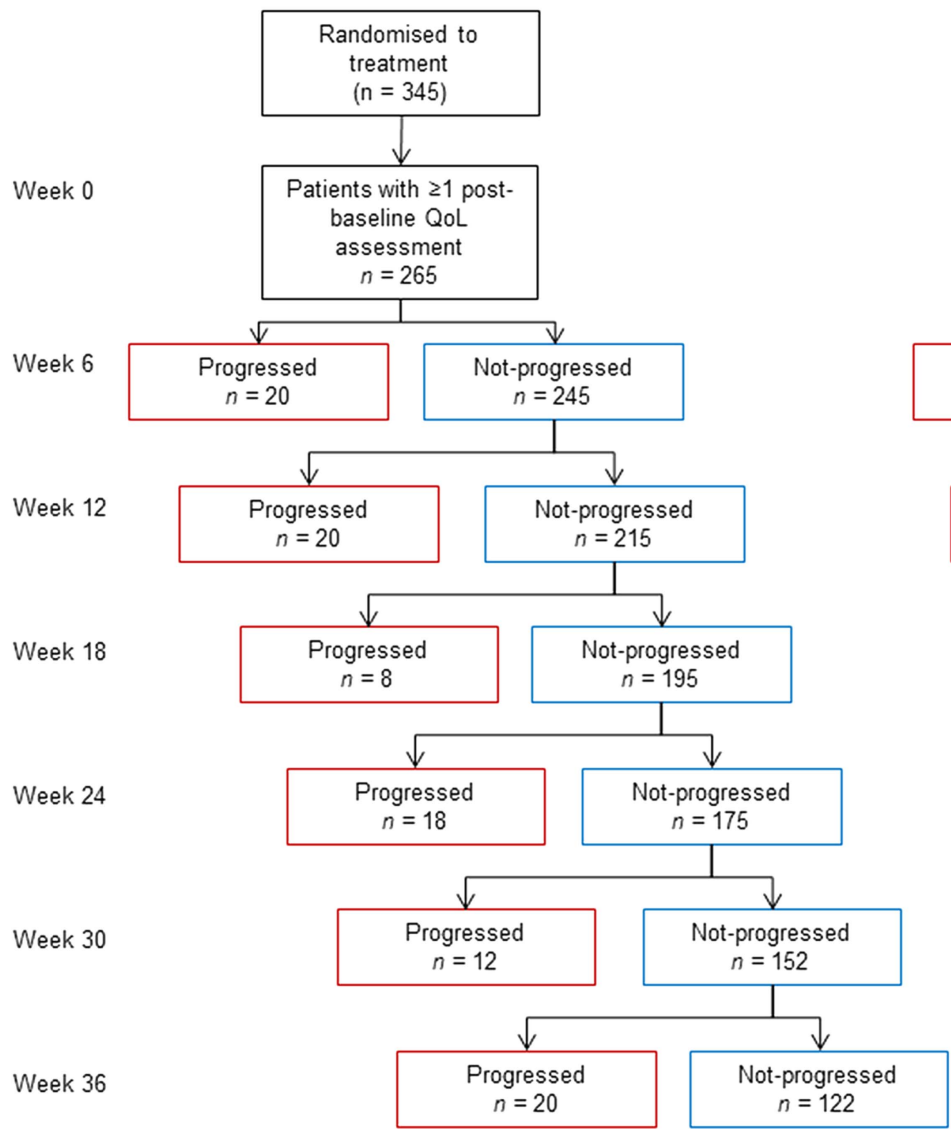

Investigator assessment

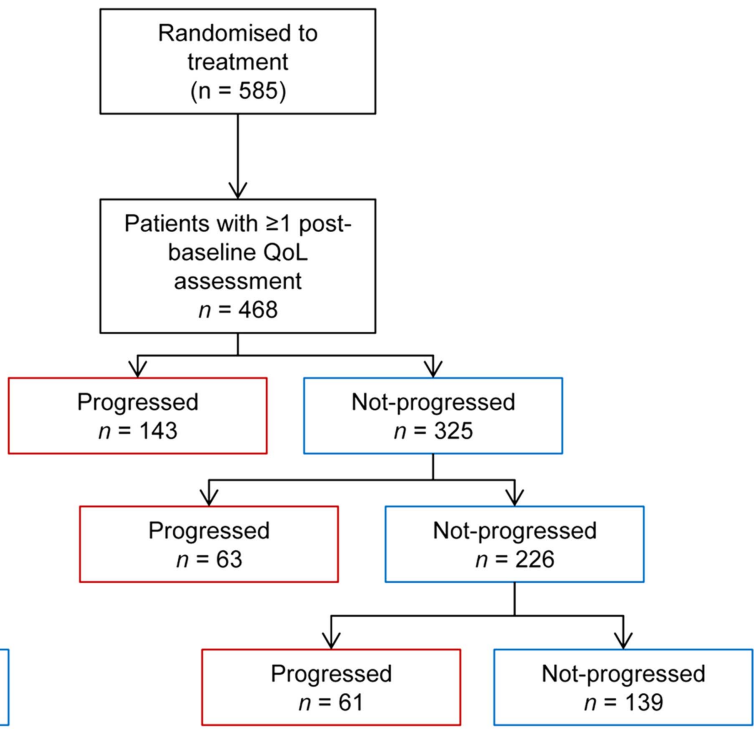

Investigator assessment

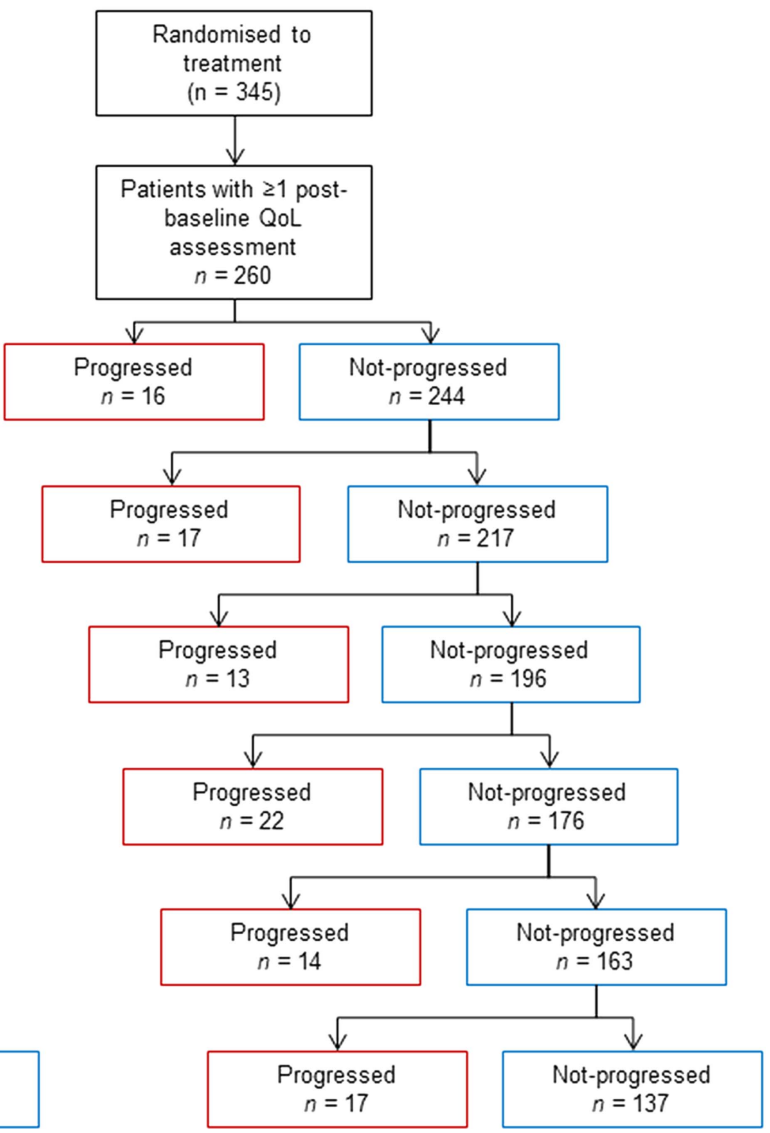

Figure 1 Flow chart of patients with disease progression by independent review and investigator assessment in LUX-Lung 1 (A) and LUX-Lung 3 (B). (QoL, quality of life). n, number of patients with progression by independent review/ investigator assessment. Patients who are censored for progression before Week 4 (LUX-Lung 1) or Week 6 (LUX-Lung 3) may not have a post-baseline HRQoL assessment; this explains the difference in the number of patients at Week 0 by each assessment method. 
related to progression status (either independent or investigator assessment) as well as baseline covariates that were used to stratify the randomisation scheme. Change in HRQoL from baseline was modelled and there was no term for randomised treatment.

\section{Model diagnostics}

For the ANCOVA as well as the longitudinal models, several model diagnostics were carried out to determine whether assumptions underlying the statistical models were valid. Normality plots of residuals and random effects, and plots of residuals against fitted values were carried out.

\section{RESULTS}

Patient population and compliance with patient-reported assessments

Patient demographics and clinical characteristics were similar between treatment arms in both trials. The numbers of patients with progression by independent review and investigator assessment at each study time point are shown in figure 1 . The difference in patient numbers between independent review and investigator assessment results from differences in censoring, death of patients prior to the first assessment, or differences in assessment of progression between independent review and investigator assessment.
LUX-Lung 1

Global health status/QoL

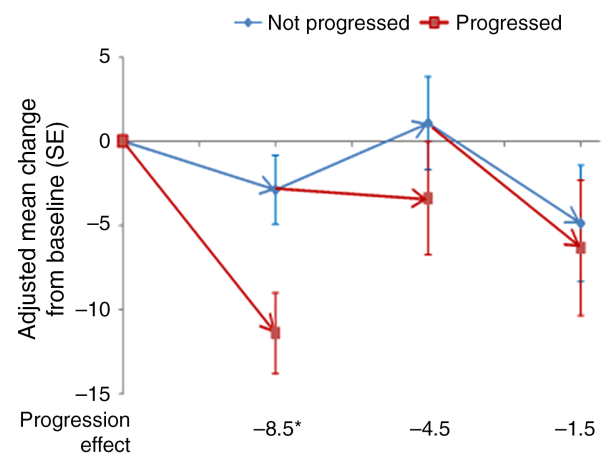

EQ-5D utility

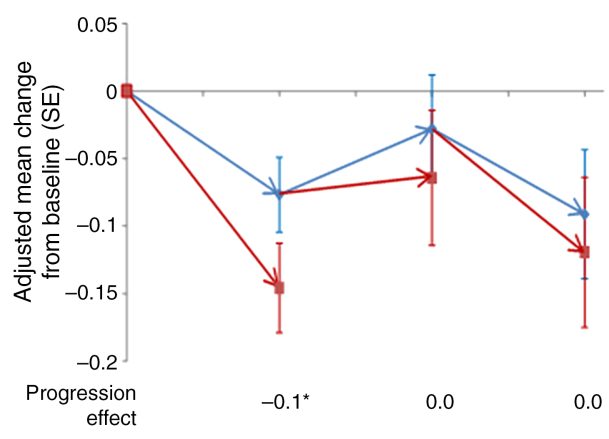

EQ-VAS

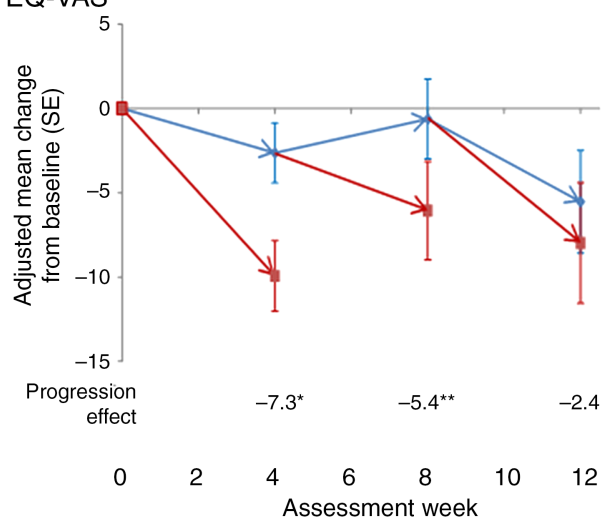

LUX-Lung 3
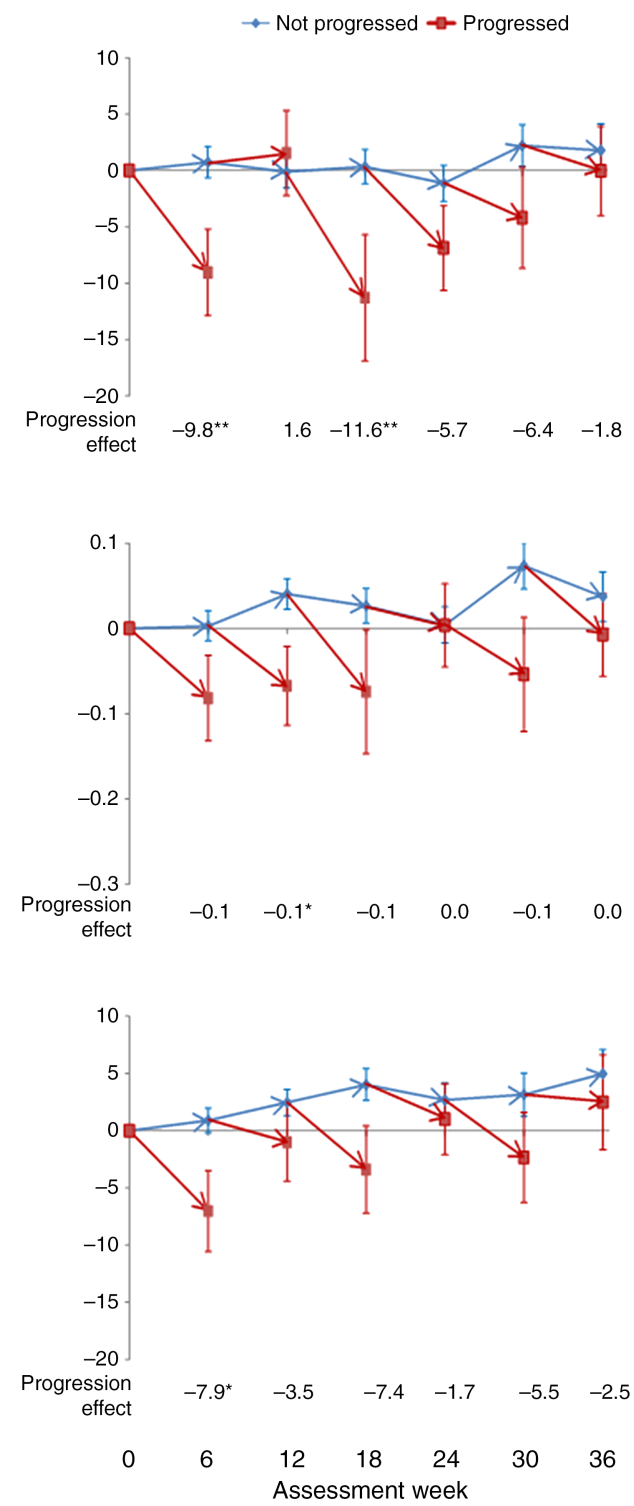

Figure 2 Progression effect and adjusted mean change from baseline for Global health status/QoL, EQ-5D utility and EQ VAS scores, by progression status in LUX-Lung 1 and LUX-Lung 3, independent review (QoL, quality of life; VAS, visual analogue scale). Progression effect: ${ }^{\star} p<0.01,{ }^{* *} p<0.05$. Arrows indicate the direction of the mean change from baseline for Global health status/QoL, EQ-5D utility and VAS scores in patients with and without progression at each assessment week. 
Baseline HRQoL questionnaires were completed by 94\% of patients in LUX-Lung 1 and $97 \%$ of patients in LUX-Lung $3 .{ }^{12}{ }^{13}$ In both studies, compliance with questionnaire completion remained high $(92-99 \%)$ at or after progression, in both treatment groups. The mean number of assessments at or after progression in patients with at least one baseline assessment was 2.5 by independent review and 1.4 by investigator assessment.

\section{Results of ANCOVA}

The progression effects (PEs) over the treatment period in LUX-Lung 1 (12 weeks) and LUX-Lung 3 (36 weeks), in terms of adjusted mean changes from baseline in EORTC Global Health/QoL, EQ-5D UK Utility and EQ VAS scores in patients with and without progression for independent review, are shown in figure 2.

In LUX-Lung 1, patients with progression by independent review consistently experienced numerically poorer HRQoL at the time of progression than patients without progression over the first 12 weeks of treatment. Mean scores differed significantly between patients with and without progression at week 4 for Global Health/ QoL (PE: -8.5), EQ-5D UK Utility (PE: -0.1) and EQ VAS scores (PE: -7.3 ; all $\mathrm{p}<0.05)$. Differences in mean change from baseline in VAS scores were also statistically significant $(p<0.05)$ between patients who experienced progression by independent review and those who did not at week 8 (PE: -5.4). Results were similar for investigator assessment of progression (see online supplementary figure 1).

In LUX-Lung 3, patients with progression by independent review consistently experienced poorer HRQoL progression than patients without progression. Differences in mean change from baseline in EORTC Global Health/QoL, EQ-5D UK Utility and EQ VAS scores were statistically significant between patients who experienced progression and those who did not at multiple time points in all assessments (figure 2). Results were similar for investigator assessment of progression (see online supplementary figure 1).
Interaction tests investigating the PE with each of baseline HRQoL and randomised treatment as well as with gender and ECOG score in LUX-Lung 1, and EGFR status and race in LUX-Lung 3 did not show any consistent trend. This indicates that these factors do not have a significant impact on the analyses and therefore supports the conclusion that the effects of progression on HRQoL that were identified by the ANCOVA models are consistent throughout the patient population.

\section{Results of the longitudinal analysis}

Estimates of the effects of progression on HRQoL from mixed-effects longitudinal models for LUX-Lung 1 and LUX-Lung 3 are shown in table 2. In both trials, progression by independent review as well as investigator assessment appeared to have consistent negative impact on all three HRQoL measures, as indicated by the negative coefficients for progression.

Estimates of the effects of progression in each treatment group separately, obtained from mixed-effects longitudinal models for LUX-Lung 1 and LUX-Lung 3, showed no significant differences between treatment groups in either study (table 3). In all analyses, PEs were consistently numerically higher when evaluated by investigator assessment than when evaluated by independent review.

\section{Model diagnostics}

Diagnostic tests confirmed that statistical methods were appropriate for the data in the two studies.

\section{DISCUSSION}

Results from the two analyses reported here suggest that tumour progression in patients with NSCLC is associated with statistically significant worsening in HRQoL, as measured using the EORTC QLQ-C30 Global Health/QoL measure and EQ-5D UK Utility and VAS scores. These findings are in agreement with previous studies in patients with breast, colorectal and renal cell cancer $^{7-10}$;

Table 2 Estimates of the effects of disease progression on HRQoL from mixed-effects longitudinal models for LUX-Lung 1 and LUX-Lung 3

\begin{tabular}{|c|c|c|c|c|}
\hline \multirow[b]{2}{*}{ HRQoL measure } & \multicolumn{2}{|l|}{ Independent review } & \multicolumn{2}{|l|}{ Investigator assessment } \\
\hline & Progression effect $(95 \% \mathrm{Cl})$ & p Value & Progression effect $(95 \% \mathrm{Cl})$ & p Value \\
\hline \multicolumn{5}{|l|}{ LUX-Lung 1} \\
\hline EORTC Global Health/QoL & $-4.24(-6.09$ to -2.40$)$ & $<0.0001$ & $-4.65(-6.44$ to -2.87$)$ & $<0.0001$ \\
\hline EQ-5D UK Utility & $-0.056(-0.083$ to -0.028$)$ & $<0.0001$ & $-0.065(-0.092$ to -0.039$)$ & $<0.0001$ \\
\hline EQ VAS & $-3.76(-5.19$ to -2.32$)$ & $<0.0001$ & $-3.83(-5.21$ to -2.44$)$ & $<0.0001$ \\
\hline \multicolumn{5}{|l|}{ LUX-Lung 3} \\
\hline EORTC Global Health/QoL & $-4.56(-6.09$ to -3.03$)$ & $<0.0001$ & $-5.62(-7.27$ to -3.96$)$ & $<0.0001$ \\
\hline EQ-5D UK Utility & $-0.061(-0.082$ to -0.041$)$ & $<0.0001$ & $-0.076(-0.099$ to -0.054$)$ & $<0.0001$ \\
\hline EQ VAS & $-3.62(-4.79$ to -2.45$)$ & $<0.0001$ & $-4.29(-5.54$ to -3.03$)$ & $<0.0001$ \\
\hline
\end{tabular}


Table 3 Effects of disease progression from mixed-effects longitudinal models for LUX-Lung 1 and LUX-Lung 3 by randomised treatment

\begin{tabular}{|c|c|c|c|c|c|c|}
\hline \multirow[b]{2}{*}{ HRQoL measure } & \multicolumn{3}{|c|}{ Independent review } & \multicolumn{3}{|c|}{ Investigator assessment } \\
\hline & \multicolumn{2}{|c|}{ Effect of progression } & \multirow[t]{2}{*}{$\begin{array}{l}\text { p Value for } \\
\text { difference } \\
\text { between } \\
\text { treatments }\end{array}$} & \multicolumn{2}{|c|}{ Effect of progression } & \multirow[t]{2}{*}{$\begin{array}{l}\text { p Value for } \\
\text { difference } \\
\text { between } \\
\text { treatments }\end{array}$} \\
\hline LUX-Lung 1 & Afatinib & Best supportive care & & Afatinib & Best supportive care & \\
\hline EORTC Global Health/QoL & -3.61 & -5.96 & 0.27 & -4.83 & -4.27 & 0.78 \\
\hline EQ-5D UK Utility & -0.060 & -0.046 & 0.66 & -0.081 & -0.033 & 0.10 \\
\hline EQ VAS & -3.63 & -4.11 & 0.77 & -4.42 & -2.55 & 0.22 \\
\hline LUX-Lung 3 & Afatinib & Cisplatin/pemetrexed & & Afatinib & Cisplatin/pemetrexed & \\
\hline EORTC Global Health/QoL & -4.65 & -4.34 & 0.85 & -5.82 & -5.15 & 0.72 \\
\hline EQ-5D UK Utility & -0.068 & -0.046 & 0.34 & -0.083 & -0.062 & 0.39 \\
\hline EQ VAS & -4.00 & -2.74 & 0.33 & -4.39 & -4.05 & 0.80 \\
\hline
\end{tabular}

A negative value indicates a deterioration in $\mathrm{HRQ}$ oL associated with progression.

$\mathrm{Cl}$, confidence interval; EORTC, European Organization for Research and Treatment of Cancer; HRQLL, health-related quality of life; VAS,

Visual Analogue Scale.

however, to our knowledge, this is the first study to show an association between tumour progression and HRQoL in patients with NSCLC.

Previous studies claiming an association between HRQoL and disease progression have been criticised for failing to apply quality assessment criteria developed to avoid potential bias when evaluating this type of association. ${ }^{4}$ Specifically, failure of analyses to censor patients at the time of progression, inadequate adjustment for confounding factors where necessary, inadequate description of whether participants were aware of their PFS status leading to potential performance bias and failure to define an a priori hypothesis regarding the relationship between PFS and HRQoL. ${ }^{4}$ In the analysis reported here, HRQoL assessments up to and beyond the time of PFS were included, confounding factors (baseline covariates) were included in the ANCOVA and analysis objectives and methods were clearly defined. In order to avoid potential performance bias, it was mandatory that patients completed HRQoL questionnaires before receiving test results, although it is possible that deviations from this may have occurred in practice.

Our results are strengthened by the use of validated assessment tools for the evaluation of HRQoL and the use of two separate analyses methods, which showed consistent findings in two trials. The longitudinal model analyses have several additional strengths over the ANCOVA, and allow for within-patient comparison of HRQoL and progression states, whereas ANCOVA only considers between-patient comparisons. The longitudinal model analyses use all available data and take appropriate account of serial correlation within a patient, resulting in more precise estimates of PE than those from ANCOVA. ${ }^{20}$ In both trials, the HRQoL assessments were comprehensive, providing a strong basis for evaluating the relationship between HRQoL and disease progression. Differences in the findings of the ANCOVA results between LUX-Lung 1 and LUX-Lung 3 may reflect the fact that clinically meaningful changes in HRQoL may be harder to achieve in heavily pretreated patients such as those included in LUX-Lung 1.

While the findings reported here indicate that disease progression is accompanied by a statistically significant worsening of HRQoL, it should also be considered whether the results represent a clinically meaningful change in HRQoL. There is continued debate as to what constitutes a meaningful change in oncology HRQoL scores, with data suggesting that patients are more responsive to improvement than decline, ${ }^{22}$ and that the thresholds for clinically significant improvement and decline are not always uniform. ${ }^{23}$ While a 10-point change in an individual patient's EORTC QLQ-C30 item or domain is an accepted threshold for clinically meaningful improvement, ${ }^{24}$ different thresholds have been proposed for intergroup changes for individual QLQ-C30 QOL scales. ${ }^{25}$ For the QLQ-C30 Global health status/QoL scale, a mean difference of 0-4 points represents a trivial effect, 4-10 point difference represents a small but clinically important effect and a 10-15 difference represents a moderate effect. ${ }^{25}$ These thresholds for QLQ-C30 Global health status/QoL imply that most of the findings reported here are clinically meaningful. For the EQ-5D UK Utility and EQ VAS scores, changes of $0.06-0.11$ and $7-12$ points, respectively, have been suggested to represent a minimally important difference, ${ }^{26}{ }^{27}$ although there is no established consensus on how best to determine the minimally important difference in HRQoL measures. ${ }^{26}$ Using these values as a guide, some of the changes observed in our study should be considered clinically meaningful.

Limitations should be considered. As more HRQoL assessments were conducted up to the time of progression, and fewer at follow-up visit(s) following progression, limited data were available on the health state of patients with progression; this is a common limitation of this type of analysis. ${ }^{4}$ Further evaluation of HRQoL in 
these patients may have revealed more pronounced differences in HRQoL between patients with and without progression. Of the two trials, LUX-Lung 3 had more HRQoL data after progression than LUX-Lung 1, indicating that the results from analysis of LUX-Lung 3 data are potentially more robust.

Accounting for, and minimising the impact of missing data (which are often not missing at random as assumed here) is an important factor in analyses such as ours. ${ }^{20}$ Full details of compliance with HRQoL questionnaires have been reported previously in the original reports of HRQoL findings from LUX-Lung $1^{11}$ and LUX-Lung $3 .{ }^{13}$ In both trials, compliance with questionnaire completion was high over the duration of treatment in each trial (LUX-Lung 1, 65-100\%; LUX-Lung 3, >90\%), which helps to reduce concern of bias due to missing data; however, attrition was greater in the control arms, with the main cause being disease progression, potentially resulting in bias. We do not consider missing data due to attrition an issue in these analyses, because we explicitly compare HRQoL in patients with and without progression at each assessment time (ANCOVA analysis), as well as assessing change in HRQoL due to progression within patients (longitudinal model); therefore, the effect of attrition should only be to reduce sample size at each assessment. Furthermore, both studies extensively evaluated the impact of missing data through sensitivity analyses and found that differences in HRQoL questionnaire completion were unlikely to bias the findings of either study.

A limitation associated with all statistical methods that estimate the effect of progression is that the comparison is non-randomised (as in an observational study) leading to potential bias. This potential bias was limited in the ANCOVA analysis by using covariate adjustment, while within patient comparisons in the longitudinal model avoided bias as long as the piecewise linear model is correct.

For ANCOVA as well as longitudinal analyses, data from active and control treatment arms were pooled, which assumes that the effect of progression on HRQoL is independent of treatment. While this may be a potential source of bias, the ANCOVA model included a term for treatment as a covariate, and estimates of treatmentspecific effects of progression from mixed-effects longitudinal models did not suggest that this was the case. It should be considered that these findings are specific to the type of patients with NSCLC enrolled in LUX-Lung 1 and LUX-Lung 3 and may not generalise to other patient types. Finally, adverse events associated with afatinib treatment have the potential to impact on specific HRQoL items $^{11} 13$ and thus have a confounding effect on the results reported here. However, there were few grade $3 / 4$ toxicities, which were confounded with assessments of progression and when these effects were included in longitudinal models the effects of progression on HRQoL were only slightly reduced (data not shown). Additionally, the HRQoL measures used in these analyses (EORTC Global Health/QoL, EQ-5D UK Utility and EQ VAS) measure global health and thus would likely reflect the effects of drug toxicity. Taking these points into consideration, we do not believe drug toxicity is an important confounding factor in our analyses.

The demonstration of a relationship between PFS and HRQoL in patients with lung cancer has important implications for healthcare policy decision-making, among others, in patients with NSCLC. It supports the use of HRQoL measures in addition to traditional clinical outcome measures such as PFS when making decisions related to patient care, and the use of PFS as proof of clinical efficacy attributable to new treatments. This is particularly relevant for new treatments that serve as add-ons to existing chemotherapy regimens, when it is often difficult to show a HRQoL benefit compared with chemotherapy alone, or where OS benefits are not shown. This analysis validates the clinical meaningfulness of PFS as a clinical trial end point.

\section{CONCLUSION}

The association between PFS and HRQoL reported here supports the use of PFS as a primary endpoint in clinical trials in patients with NSCLC, as it confirms the value of PFS as a patient-relevant endpoint associated with tangible improvements in HRQoL.

Acknowledgements Medical writing assistance, supported financially by Boehringer Ingelheim, was provided by Suzanne Patel during the preparation of this article.

Contributors IG, MP, PMF and SE contributed to the conception and design of the analysis. MP, PMF and SE conducted the analyses and all authors contributed to the interpretation of data. The first draft of the manuscript was written by IG. All authors contributed to critically revising the text for important intellectual content. All authors provided final approval of this submitted version.

Funding This study was supported by Boehringer Ingelheim.

Competing interests MP, PMF and SE have received consultancy fees from Boehringer Ingelheim. IG is an employee of Boehringer Ingelheim.

Provenance and peer review Not commissioned; externally peer reviewed.

Data sharing statement No additional data are available.

Open Access This is an Open Access article distributed in accordance with the Creative Commons Attribution Non Commercial (CC BY-NC 4.0) license, which permits others to distribute, remix, adapt, build upon this work noncommercially, and license their derivative works on different terms, provided the original work is properly cited and the use is non-commercial. See: http:// creativecommons.org/licenses/by-nc/4.0/

\section{REFERENCES}

1. Fallowfield LJ, Fleissig A. The value of progression-free survival to patients with advanced-stage cancer. Nat Rev Clin Oncol 2012;9:41-7.

2. Soria JC, Massard C, Le Chevalier T. Should progression-free survival be the primary measure of efficacy for advanced NSCLC therapy? Ann Oncol 2010;21:2324-32.

3. Villaruz LC, Socinski MA. The clinical viewpoint: definitions, limitations of RECIST, practical considerations of measurement. Clin Cancer Res 2013;19:2629-36.

4. Gutman SI, Piper M, Grant MD, et al. Progression-free survival: what does it mean for psychological well-being or quality of life? AHRQ methods for effective health care. 2013; Report No.: 13-EHC074-EF. Published Online First. 
5. Beckman M. More clinical cancer treatments judged by progressionfree rather than overall survival. J Natl Cancer Inst 2007;99:1068-9.

6. Institut für Wirtschaftlichkeit und Qualität im Gesundheitswesen. Aussagekraft von Surrogatendpunkten in der Onkologie. 2011;

Rapid Report 1.1 Nr. 80. Published Online First. http://www.iqwig.de

7. Zhou X, Cella D, Cameron D, et al. Lapatinib plus capecitabine versus capecitabine alone for HER2+ (ErbB2+) metastatic breast cancer: quality-of-life assessment. Breast Cancer Res Treat 2009;117:577-89.

8. Sherrill B, Amonkar MM, Sherif B, et al. Quality of life in hormone receptor-positive HER-2+ metastatic breast cancer patients during treatment with letrozole alone or in combination with lapatinib. Oncologist 2010;15:944-53.

9. Siena S, Peeters M, Van Cutsem E, et al. Association of progression-free survival with patient-reported outcomes and survival: results from a randomised phase 3 trial of panitumumab. Br J Cancer 2007;97:1469-74.

10. Cella D, Pickard AS, Duh MS, et al. Health-related quality of life in patients with advanced renal cell carcinoma receiving pazopanib or placebo in a randomised phase III trial. Eur J Cancer 2012;48:311-23.

11. Hirsh V, Cadranel J, Cong XJ, et al. Symptom and quality of life benefit of afatinib in advanced non-small-cell lung cancer patients previously treated with erlotinib or gefitinib: results of a randomized phase Ilb/III trial (LUX-Lung 1). J Thorac Oncol 2013;8:229-37.

12. Miller VA, Hirsh V, Cadranel J, et al. Afatinib versus placebo for patients with advanced, metastatic non-small-cell lung cancer after failure of erlotinib, gefitinib, or both, and one or two lines of chemotherapy (LUX-Lung 1): a phase $2 \mathrm{~b} / 3$ randomised trial. Lancet Oncol 2012;13:528-38.

13. Yang JC, Hirsh V, Schuler M, et al. Symptom control and quality of life in LUX-Lung 3: a phase III study of afatinib or cisplatin/ pemetrexed in patients with advanced lung adenocarcinoma with EGFR mutations. J Clin Oncol 2013;31:3342-50.

14. Sequist LV, Yang JC, Yamamoto N, et al. Phase III study of afatinib or cisplatin plus pemetrexed in patients with metastatic lung adenocarcinoma with EGFR mutations. J Clin Oncol 2013;31:3327-34

15. Aaronson NK, Ahmedzai S, Bergman B, et al. The European Organization for Research and Treatment of Cancer QLQ-C30: a quality-of-life instrument for use in international clinical trials in oncology. J Natl Cancer Inst 1993;85:365-76.

16. EuroQol G. EuroQol-a new facility for the measurement of health-related quality of life. Health Policy 1990;16:199-208.

17. Dolan P. Modeling valuations for EuroQol health states. Med Care 1997;35:1095-108.

18. Eisenhauer EA, Therasse $\mathrm{P}$, Bogaerts $\mathrm{J}$, et al. New response evaluation criteria in solid tumours: revised RECIST guideline (version 1.1). Eur J Cancer 2009;45:228-47.

19. Therasse $P$, Arbuck SG, Eisenhauer EA, et al. New guidelines to evaluate the response to treatment in solid tumors. European Organization for Research and Treatment of Cancer, National Cancer Institute of the United States, National Cancer Institute of Canada. J Natl Cancer Inst 2000;92:205-16.

20. Fairclough DL. Design and analysis of quality of life studies in clinical trials. 2nd edn. London: Chapman \& Hall, 2010.

21. Diggle PJ, Heagerty P, Liang K-Y, et al. Analysis of longitudinal data. Oxford: Clarendon, 2002.

22. Bedard G, Zeng L, Lam H, et al. Meaningful change in oncology quality-of-life instruments: a systematic literature review. Expert Rev Pharmacoecon Outcomes Res 2012;12:475-83.

23. Cocks K, King MT, Velikova G, et al. Evidence-based guidelines for interpreting change scores for the European Organisation for the Research and Treatment of Cancer Quality of Life Questionnaire Core 30. Eur J Cancer 2012;48:1713-21.

24. Osoba D, Rodrigues G, Myles J, et al. Interpreting the significance of changes in health-related quality-of-life scores. J Clin Oncol 1998;16:139-44.

25. Cocks K, King MT, Velikova G, et al. Evidence-based guidelines for determination of sample size and interpretation of the European Organisation for the Research and Treatment of Cancer Quality of Life Questionnaire Core 30. J Clin Oncol 2011;29:89-96.

26. Luo N, Johnson J, Coons SJ. Using instrument-defined health state transitions to estimate minimally important differences for four preference-based health-related quality of life instruments. Med Care 2010;48:365-71.

27. Pickard AS, Neary MP, Cella D. Estimation of minimally important differences in EQ-5D utility and VAS scores in cancer. Health Qual Life Outcomes 2007;5:70. 